\title{
reviscafuences
}

ISSN: 1575-7072 | e-ISSN: 2172-7775

Páginas: $24-36$

Recibido: 2018-12-10

Revisado: 2019-08-12

Aceptado: 2019-11-24

Preprint: 2020-04-20

Publicación Final: 2020-06-15

www.revistascientificas.us.es/index.php/fuentes/index

DOI: https://doi.org/10.12795/revistafuentes.2020.v22.11.03

\section{Comparaciones múltiples de dificultades emocionales y de comportamiento, procesos de comunicación, vocabulario receptivo y razonamiento abstracto en niños y adolescentes con dispositivos auditivos en Islas Canarias}

\section{Multiple comparisons of emotional and behavioural difficulties, communication processes, receptive vocabulary and abstract reasoning in children and adolescents with hearing devices in Islas Canarias}

\section{(iD) Olga María Alegre de la Rosa}

Universidad de La Laguna (España)

\section{Resumen}

El presente estudio consideró que los niños y adolescentes que usan dispositivos auditivos manifiestan dificultades emocionales y de comportamiento, entre otras complicaciones psicológicas. El estudio tuvo como objetivo investigar si las dificultades emocionales y de comportamiento, los procesos de comunicación, el vocabulario receptivo y el razonamiento abstracto diferían entre los niños y adolescentes que usaban implantes cocleares (ICs) o audífonos (AUDs), de acuerdo con la evaluación de múltiples informantes. Método. Se correlacionaron los resultados de los niños y adolescentes con dificultades auditivas en cuatro cuestionarios y pruebas psicológicas (SDQ, ITPA, Peabody y Raven) y se identificaron nuevos predictores de las dificultades emocionales y de comportamiento de dichos niños y adolescentes. El estudio involucró a 187 niños y adolescentes con ICs, 113 con AUDs de las dos provincias de las Islas Canarias. También participaron 176 padres y madres y 300 profesores. Resultados. Se hallaron desacuerdos y diferencias significativas entre los múltiples informantes (niños y adolescentes, familias y profesorado) en términos de sus evaluaciones de SDQ. La puntuación total de SDQ se correlacionó ligeramente con otros resultados finales de Peabody y Raven. El análisis de regresión lineal mostró seis variables que predijeron significativamente la puntuación total en el SDQ de los niños y adolescentes que usan dispositivos auditivos. Conclusiones. Los hallazgos tienen consecuencias para el desarrollo de programas formativos del profesorado de educación primaria y secundaria, así como en los sistemas evaluativos relacionados con los aspectos emocionales y de comportamiento, los procesos de comunicación, el vocabulario receptivo y el razonamiento abstracto empleados con los niños y adolescentes que usan dispositivos auditivos en Islas Canarias.

\section{Abstract}

The present study considered that children and adolescents with hearing devices keep emotional and behavioural difficulties, among other psychological complications. The study aimed to investigate whether emotional and behavioural difficulties, communication processes, receptive vocabulary and abstract reasoning differed between children and adolescents with cochlear implants (Cls) or hearing aids (HAs), according to multi-informant ratings. Method. The results of children and adolescents with hearing impairments were correlated in four questionnaires and psychological tests (SDQ, ITPA, Peabody and Raven) and to identify new socio-demographic and linguistic predictors of emotional problems and difficulties in children and adolescents with hearing devices. The study involved 187 children and adolescents with Cls, 113 children and adolescents with HAs from the two provinces of Canary Islands. 176 fathers and mothers, and 300 schoolteachers also participated. Results. Perception disagreements were found between children and adolescents, families and schoolteachers in terms of their SDQ ratings with significant differences among the multi-informant. Total SDQ score lowly correlated with other total ITPA, and final Peabody and Raven outcomes. Regression linear analysis showed six socio-demographic and linguistic covariates that predicted significantly children and adolescents who used hearing devices in the total SDQ score. Conclusion. Findings have consequences for developing schoolteacher-training programs of primary and secondary education and implementing evaluation systems related to the emotional and behavioural difficulties, communication processes, receptive vocabulary and abstract reasoning used with children and adolescents with hearing devices in Islas Canarias.

\section{Palabras clave / Keywords}

Dispositivos auditivos, implante coclear, audífono, familia, profesorado, SDQ

Hearing devices, cochlear implantation, hearing aid, family, schoolteacher, SDQ 


\section{Introducción}

El estudio de las dificultades emocionales y de comportamiento, los procesos de comunicación, el vocabulario receptivo y el razonamiento abstracto en niños y adolescentes con dispositivos auditivos (implantes cocleares (ICs) o audífonos (AUDs) adquiere relevancia en el sistema educativo canario por su incidencia en el desarrollo integral del estudiante. La identificación de diferencias psicológicas entre niños y adolescentes con dispositivos auditivos plantea problemas que abordamos en el presente estudio.

\subsection{Mediciones de las diferencias individuales de niños y adolescentes con implantes auditivos}

Una de las líneas de investigación desarrollada para medir las diferencias individuales es la evaluación de las dificultades emocionales y de comportamiento de los niños y adolescentes con disminución auditiva relacionada con las estructuras mentales del lenguaje (Henner, Novogrodsky, Reis y Hoffmeister, 2018). El conocimiento de la información sensorial percibida por los niños y adolescentes con dispositivos auditivos es esencial para superar el retraso que tienen en el lenguaje hablado. Muchas pruebas diagnósticas exigen conocimientos lingüísticos a los niños y adolescentes con dificultades auditivas. Sin embargo, la aplicación de algunas pruebas preocupa a los investigadores debido a la dificultad de comprensión de las instrucciones de empleo por el desarrollo lingüístico insuficiente de los niños y adolescentes con disminución auditiva. En efecto, las dificultades de los niños y adolescentes con ICs o AUDs afectan su rendimiento, adaptación inteligente y calidad de vida (Edwards, Hill, y Mahon, 2012).

Conocer las dificultades emocionales y de comportamiento en términos de fortalezas y debilidades personales en niños y adolescentes que emplean dispositivos auditivos es un objetivo claro en los estudios que han empleado el Cuestionario de Fortalezas y Dificultades (SDQ, por sus siglas en inglés), el cual se concibe como un instrumento para cribar, entre otras funciones, el estado de salud mental niños y adolescentes de 4 a 16 años (Goodman, 1997). La estructura y fiabilidad de SDQ están comprobadas en muchos países, incluida España (Ortuño-Sierra, Aritio-Solana, y Fonseca-Pedrero, 2018; Rodríguez-Hernández et al., 2014). Este cuestionario se ha aplicado solo o conjuntamente con otras pruebas de comunicación y de habilidad lingüística para informar a las familias y al personal educativo de los centros escolares del estado mental de niños y adolescentes. En este sentido, Huber y Kipman (2011) utilizaron el SDQ para evaluar el estado de salud mental de 32 adolescentes con ICs y 212 compañeros con audición típica. A partir de su estudio, extrajeron una conclusión: "Los docentes calificaron a los niños que tenían ICs con más problemas con sus compañeros y más problemas "totales" (p. 152). En otro trabajo, Wong et al. (2017, p. 15) encontraron que "los niños con ICs o AUDs no mostraron dificultades significativas emocionales o de comportamiento en comparación con los de audición típica (SDQ)". Posteriormente, Wong et al. (2018) compararon las percepciones de las familias y los educadores sobre el comportamiento social de niños con ICs utilizando SDQ y otros instrumentos averiguando que los padres necesitaban ayuda para resolver problemas comportamentales en casa y que los profesores deberían dar más oportunidades para practicar la conducta prosocial en la escuela.

Ocurre con frecuencia una variación en los resultados de los procesos de comunicación de niños y adolescentes después de la implantación de dispositivos auditivos. La evaluación específica de todas las habilidades de comunicación de los niños y adolescentes con ICs o AUDs despierta un interés de investigación cuando se usa ITPA. Test Illinois de habilidades psicolingüísticas, o de alguna de las subpruebas que lo componen (p.ej., fluidez lingüística, o comprensión de frases o cuentos). Sin embargo, ITPA es una medida para determinar la formación psicolingüística específica de educadores, dada la variedad de habilidades cognitivas diagnosticadas de los niños (Kirk y Elkins, 1974). En este sentido, el estudio de Hassan, Eeldin y Al Kasaby (2014) determinó el perfil de las habilidades psicolingüísticas de los niños de habla árabe con pérdidas auditivas.

El vocabulario receptivo forma parte del lexicón mental de un hablante, es decir, el conocimiento que un hablante tiene interiorizado del vocabulario. El Peabody, Test de Vocabulario en Imágenes (PPVT, abreviado en inglés) permite estudiar el vocabulario de sujetos con dificultades auditivas y determina cómo la edad de la implantación coclear es un predictor esencial de los resultados del habla y del lenguaje de niños y adolescentes con ICs (Connor, Craig, Raudenbush, Heavner, y Zwolan, 2006). Otros investigadores han explorado el grado en que los niños y adolescentes con pérdida auditiva unilateral y leve bilateral corren el riesgo de tener dificultades con habilidades sociales en comparación con los que tienen audición típica, o con aquellos con pérdida auditiva moderada a grave (Laugen, Jacobsen, Rieffe y Wichstrøm, 2017). En fin, Peabody permite conocer los resultados del retraso en el vocabulario receptivo y documentar otros factores predictores del desarrollo del lenguaje en niños con ICs (Sarchet et al., 2014; Sarant, Harris, Bennet, y Bant, 2014). 
Los niños y adolescentes con dispositivos auditivos muestran una comprensión más lenta de la audición y del lenguaje hablado. La aplicación del Test de matrices progresivas de Raven (SPM, abreviado en inglés) estima la fluidez del razonamiento abstracto de los niños con ICs y AUDs. Debido al elevado número de ítems del test de Raven, algunos investigadores han reducido el tamaño del test (Bilker et al., 2012), sin perder su validez predictiva, demostrando que "los elementos son en gran medida invariantes" (Lúcio, Cogo-Moreira, Puglisi, Polanczyk, y Little, 2017, p. 9). Después de la aplicación del test Raven de inteligencia (AskerArnason, Ibertsson, Wass, Wengelin y Sahlen, 2010) se han conocido las limitaciones del procesamiento lingüístico y cognitivo de niños con ICs en comparación con compañeros que escuchan tareas semánticas complejas. Los niños con ICs perciben música alterada debido a sus dificultades auditivas. Shirvani y otros (2016) utilizaron la versión iraní de 32 elementos del test de Raven para comparar tres grupos de niños (audición típica, ajuste bimodal y pérdida auditiva profunda y con IC unilateral), y encontraron que "una débil percepción de las emociones musicales en niños con IC unilateral puede llevar a una debilidad en el mensaje comunicativo de la música" (pp. 475-476).

\subsection{Significado y objetivos del estudio}

Los padres y las madres, así como el profesorado, se enfrentan a muchos desafíos para tomar decisiones relacionadas con las dificultades emocionales y de comportamiento, crear opiniones informadas sobre los procesos de comunicación, estimular vocabularios receptivos, inducir razonamientos abstractos y promover el desarrollo del lenguaje hablado o de signos en niños y adolescentes con dificultades auditivas (Isarin et al., 2015; Wong et al., 2018). La resolución de estas necesidades educativas sustenta los objetivos de la presente investigación:

Primero, investigar las dificultades emocionales y de comportamiento de niños y adolescentes con dificultades auditivas después de administrar el cuestionario SDQ, y constatar la homogeneidad evaluativa de múltiples informantes (niños y adolescentes, familias y profesorado). El cálculo del acuerdo entre informantes de los problemas y dificultades de niños y adolescentes con ICs y AUDs en un esfuerzo metodológico orientado a dar homogeneidad y consistencia a las puntuaciones del cuestionario SDQ. Así han procedido investigadores para constar las competencias de aprendizaje socio-emotivas cuando se usan escalas de comportamiento (Gresham et al., 2017).

Segundo, descubrir las relaciones funcionales de las puntuaciones totales en las dificultades emocionales y de comportamiento (SDQ) y las puntuaciones totales en los procesos de comunicación (ITPA), vocabulario receptivo (PPVT) y razonamiento abstracto (SPM) de niños y adolescentes con dificultades auditivas. Investigaciones de relaciones entre variables usando alguno de los instrumentos seleccionados para este estudio aparecen en Sarchet et al. (2014).

Tercero, revelar las relaciones de efecto causal entre variables. Concretamente, predecir la puntuación total de las dificultades emocionales y de comportamiento (SDQ) según las puntuaciones totales en los procesos de comunicación (ITPA), vocabulario receptivo (PPVT) y razonamiento abstracto (SPM) de niños y adolescentes con dificultades auditivas. De manera similar, calcular la puntuación total de las dificultades emocionales y de comportamiento (SDQ) de los niños y adolescentes con dispositivos auditivos, según variables sociodemográficas de los informantes: género, dispositivo auditivo, adaptación curricular individualizada $(\mathrm{ACl})$, edad cronológica y de implantación; nivel escolar, grado, terapia del habla en la escuela (número de horas), experiencia del profesorado (número de años); estudios de la familia, edad de los padres y tipo de comunicación doméstica, ubicación geográfica, entre otras variables. Así han procedido autores que han estudiado las dificultades en el lenguaje de adolescentes, realizando predicciones de sus diferencias individuales en conductas sociales y en la habilidad lingüística (Durkin y Conti-Ramsden, 2007).

\section{Método}

\subsection{Participantes}

La muestra del estudio consistió en niños y adolescentes con ICs $(n=187)$, sin otros déficits asociados, residentes en las islas Canarias. Se seleccionaron, a su vez, niños y adolescentes con AUDs $(n=113)$ que no tuvieran tampoco otros déficits asociados. Del total de la muestra, 137 fueron de la provincia de Tenerife y 163 de la provincia de Gran Canaria. Las edades fueron entre 6-9 años $(n=104), 10-12$ años $(n=88)$ y 1316 años $(n=108)$. Asistían a centros educativos ordinarios. De ellos, 119 siguieron un programa de Adaptación Curricular Individualizada ( $\mathrm{ACl})$, mientras que 181 estudiantes cursaron el mismo programa curricular que sus compañeros oyentes. 
Se obtuvo un consentimiento por escrito de los padres o madres para administrar pruebas a cada niño o adolescente. Además, se obtuvo la aprobación de las disposiciones éticas y legales del Comité de Ética de Investigación Clínica del Hospital Universitario Insular Materno-Infantil de Canarias. El papel de la familia era esencial para la comunicación de los niños y adolescentes (Ching et al., 2018), por tanto, incluimos la formación y estado laboral de los padres como variables que podrían predecir fortalezas y problemas psicoeducativos de los niños y adolescentes (Porter et al., 2013). Los padres y madres tenían una formación que iba desde Educación Primaria a Educación Superior, siendo la Educación Primaria, la mayoritaria (padres $n=87$ y madres $n=89$ ). Aunque algunos padres estaban en paro o eran jubilados, la mayoría estaba en situación laboral activa (padres: $n=230$, madres: $n=213$ ). De otra parte, las edades mayoritarias de los padres abarcó la horquilla de 41 y 50 años, tanto para los padres como para las madres $(n=143)$.

Los padres que utilizaban comunicación oral $(n=215)$ fueron mayoría, en comparación con los que solo empleaban signos $(n=28)$ o eran bilingües $(n=57)$.

Un estudio canadiense señaló que los profesores tutores informaban de actitudes adecuadas para la inclusión de niños con pérdida auditiva en sus aulas (Eriks-Brophy y Whittingham, 2013), mientras que un estudio estadounidense manifestó que los terapeutas (equivalentes a profesores especialistas en audición y lenguaje) no habían sido preparados para gestionar la tecnología de los implantes cocleares y otros temas auditivos (Compton, Tucker y Flynn, 2009). Incorporamos a los profesores tutores y a los especialistas en audición y lenguaje de los niños y adolescentes en el presente estudio para conocer el índice de acuerdo de sus puntuaciones a la hora de considerar las dificultades emocionales y de comportamiento, los procesos de comunicación, el vocabulario receptivo y el razonamiento abstracto de los niños y adolescentes con ICs o AUDs.

Los especialistas en audición y lenguaje fueron mayoritariamente mujeres $(n=249)$ y también las profesoras tutoras $(n=227)$, en comparación con los profesores varones: especialistas en audición y lenguaje $(n=51)$ y profesores tutores $(n=73)$. La mayoría de los especialistas tenían entre 50 y 59 años $(n=171)$, y la edad de los profesores tutores era entre 60 o más años $(n=150)$. En promedio, ambos tipos de educadores escolares tenían diez años o más de experiencia profesional: especialistas $(n=193)$ y tutores $(n=185)$.

\subsection{Instrumentos}

Cuestionario de Fortalezas y Dificultades (SDQ). Goodman $(1997,2001)$ diseñó y desarrolló el Cuestionario de Fortalezas y Dificultades (SDQ) que consta de 25 ítems agrupados en cinco escalas: problemas emocionales, problemas de conducta, problemas con compañeros, hiperactividad y comportamiento prosocial. EI SDQ es una herramienta breve que permite filtrar el estado mental de niños y adolescentes 4 a 16 años. Ortuño-Sierra, Fonseca-Pedrero, Inchausti, y Sastre i Riba (2016) han sintetizado artículos nacionales y extranjeros que refieren las propiedades psicométricas del cuestionario. Los valores alfa de Cronbach son diferentes en el presente estudio según los grupos estudiados: niños (0.789), especialistas (0.725), tutores (0.616) y familias (padres y madres) (0.759), que representaron un valor adecuado de fiabilidad (George y Mallery, 2003).

ITPA. Test Illinois de habilidades psicolingüísticas (Kirk, McCarthy y Kirk, 2001). Antes de la versión traducida de ITPA al español, 68 estudios adoptaron este instrumento entre 1968 y 1974 para investigar las habilidades de los niños y adolescentes (Kirk y Elkins, 1974). Está compuesto por diez áreas (comprensión auditiva, comprensión visual, memoria secuencial visual-motora, asociación auditiva, memoria secuencial auditiva, asociación visual, integración visual, expresión verbal e integración gramatical). Permite la detección, predicción y diagnóstico de posibles errores o dificultades en el proceso de comunicación. Algunos investigadores aplicaron todo el instrumento a niños y adolescentes con ICs (Jiménez, Pino y Herruzo, 2009) o algunas subpruebas para predecir las diferencias individuales en la aceptación de los compañeros y la formación de amistad entre los niños con discapacidades específicas del lenguaje (Durkin y Conti-Ramsden, 2007). El valor alfa de Cronbach para este estudio fue de 0.852 , que significó un coeficiente de confiabilidad razonable (George y Mallery, 2003).

Peabody. Test de Vocabulario en Imágenes (PPVT). Esta prueba (PPVT-4, Dunn y Dunn, 2007) contiene una evaluación de vocabulario receptivo que ofrece dos formas alternativas. Cada formulario consta de 228 elementos, agrupados en 19 conjuntos de 12 elementos que aumentan en dificultad, que se aplica, según la edad del niño o su nivel de habilidad, limitando el tiempo de administración (10-15 minutos). Los estudiantes señalan una de las cuatro imágenes que mejor representan el significado de una palabra estímulo ofrecida verbalmente. El coeficiente de confiabilidad promedio para esta prueba fue de 0.89 (Sarant, Harris, Bennet y Bant, 2014). El resultado del valor alfa de Cronbach para este estudio fue de 0.641 , que se consideró un coeficiente cuestionable (George \& Mallery, 2003). 
Test de matrices progresivas de Raven (SPM) (Raven, 1989, 2000). Este instrumento se ha distribuido a niños y adolescentes con dificultades auditivas durante los últimos 50 años (Henner, Novogrodsky, Reis y Hoffmeister, 2018). Comprende 60 elementos que miden el razonamiento abstracto donde los niños y adolescentes deben seleccionar el fragmento ausente de un plan incompleto. En la base de esta construcción se encuentra la recopilación de datos de matrices progresivas. El resultado del alfa de Cronbach para este estudio fue 0.713 , que pareció un coeficiente aceptable (George y Mallery, 2003).

\subsection{Procedimiento}

Se obtuvo el consentimiento por escrito de cada padre, madre o ambos, para participar en este estudio. Los padres, profesores, niños y adolescentes con dispositivos auditivos completaron SDQ y un cuestionario de datos sociodemográficos. Dicho cuestionario buscó información sobre género, pérdida auditiva de los niños y adolescentes, modo de comunicación, ubicación geográfica y experiencia educativa.

La situación económica se midió a partir del empleo de las familias. Logopedas entrenados administraron las pruebas ITPA, Peabody y Raven. Cada sesión de prueba duró una mañana con las pausas apropiadas.

\subsection{Análisis de datos}

Primero se calcularon las estadísticas descriptivas. Segundo, se calculó la $k$ de Cohen entre niños y adolescentes, tutores y especialistas, y profesorado y miembros de las familias en las subescalas SDQ para conocer el nivel de acuerdo en las puntuaciones. Tercero, los coeficientes de correlación de Pearson examinaron la fuerza y la dirección de la relación lineal entre las respuestas de los niños y adolescentes en las subescalas SDQ y las puntuaciones totales de ITPA y las puntuaciones finales de Peabody y Raven. Cuarto, se realizó una correlación de Pearson entre el profesorado y los niños y adolescentes sobre las puntuaciones totales de SDQ, las puntuaciones totales de ITPA y las puntuaciones finales de Peabody y Raven. Quinto, se realizó un análisis de regresión múltiple para examinar el poder predictivo de variables independientes en la puntuación total del SDQ. Las variables independientes fueron los datos sociodemográficos de los participantes y variables de las áreas de ITPA. Todos los análisis se realizaron con la versión 21 de SPSS.

\section{Resultados}

\subsection{Examen del acuerdo entre evaluadores de las subescalas SDQ, mediante kappa de Cohen.}

De acuerdo con el primer objetivo, se encontró concordancia leve entre los profesores tutores y profesores especialistas en las puntuaciones de las subescalas del SDQ de los niños y adolescentes con dispositivos auditivos. Además, se reveló un acuerdo moderado entre ambos profesionales en la subescala Problemas de conducta $(k=-.405, p<.001)$ para el caso de los que estudiantes que utilizaban audífonos.

Se encontró un acuerdo moderado entre los niños y adolescentes y sus profesores especialistas en la subescala Problemas de conducta del SDQ total en los niños y adolescentes con AUDs $(k=.415, p<.001)$. De manera similar, se descubrió un acuerdo moderado en la subescala de Problemas emocionales de los niños y adolescentes con ICs y sus tutores $(k=.410, p<.001)$.

\subsection{Relaciones entre las cinco subescalas de SDQ y las áreas, conjuntos o elementos de ITPA, PPVT y RPM.}

La Tabla 1 proporciona relaciones bajas, aunque significativas entre las puntuaciones de las subescalas SDQ de los niños y adolescentes (p. ej., Problemas emocionales correlacionados con Problemas de conducta $(r=$ $.325, p<.001)$ y correlaciones bajas y negativas con Problemas con compañeros $(r=-.122, p<.05)$, lo que significa que cuando los Problemas emocionales aumentaron, los Problemas con los compañeros disminuyeron en una proporción constante. Los análisis de correlación indicaron que los Problemas de conducta demostraron correlaciones significativas y bajas con Problemas con compañeros $(r=.391, p<.001)$, Hiperactividad $(r=.260, p<.001)$ y correlaciones negativas y pequeñas con Conducta prosocial $(r=-.298, p$ $<.001$ ), lo que significa que cuando aumentaron los Problemas de conducta, la Conducta prosocial disminuyó en una proporción constante. Además, los coeficientes de correlación indicaron una relación significativa y pequeña entre Hiperactividad y Problemas con compañeros $(r=.445, p<.001)$, y correspondencias negativas y pequeñas con Conducta prosocial $(r=-.291, p<.001)$, significando que cuando la Hiperactividad aumentaba, la Conducta prosocial disminuía en una proporción constante. Finalmente, la subescala Problemas con compañeros se correlacionó de manera significativa y negativa con la subescala Conducta prosocial $(r=-$ 
$.432, p<.001)$, que significaba que cuando los Problemas con los compañeros aumentaban, la Conducta prosocial disminuía en una proporción constante.

Además, se encontró una correlación estadísticamente significativa, baja y positiva entre las puntuaciones de las subescalas de SDQ, la puntuación total de ITPA $(r=.115, p<.05)$ y la puntuación final de RPM $(r=.115$, $p<.05)$. Los hallazgos también revelaron que la puntuación total de ITPA fue baja y se correlacionó con la puntuación final de RPM $(r=.115, p<.05)$ y con algunas puntuaciones de las subescalas de SDQ (p. ej., Problemas de conducta $(r=.163 p<.001)$, e Hiperactividad $(r=.177, p<.001)$. Además, se encontraron correlaciones significativas y bajas entre la puntuación final de PPVT con puntuaciones de subescalas de SDQ (p. ej., Problemas de conducta $(r=.118, p<.05)$ y Conducta prosocial $(r=-.141, p<.05)$.

Tabla 1

Correlaciones entre las cinco subescalas de SDQ e ITPA, PPVT y RPM para niños y adolescentes con dispositivos auditivos

\begin{tabular}{|c|c|c|c|c|c|c|c|c|}
\hline & ITPA & PPVT & RPM & $\begin{array}{l}\text { Problemas } \\
\text { emocionales }\end{array}$ & $\begin{array}{c}\text { Problemas } \\
\text { conducta }\end{array}$ & Hiperactividad & $\begin{array}{l}\text { Problemas con } \\
\text { compañeros }\end{array}$ & $\begin{array}{l}\text { Conducta } \\
\text { prosocial }\end{array}$ \\
\hline ITPA & 1.000 & & & & & & & \\
\hline \multirow{2}{*}{ PPVT } & -.073 & 1.000 & & & & & & \\
\hline & .206 & & & & & & & \\
\hline \multirow{2}{*}{ RPM } & $.115^{*}$ & .077 & 1.000 & & & & & \\
\hline & .046 & .186 & & & & & & \\
\hline \multirow{2}{*}{$\begin{array}{l}\text { Problemas } \\
\text { emocionales }\end{array}$} & -.049 & -.053 & .016 & 1.000 & & & & \\
\hline & .394 & .358 & .781 & & .000 & & & \\
\hline \multirow{2}{*}{$\begin{array}{l}\text { Problemas } \\
\text { conducta }\end{array}$} & $-.163^{* *}$ & $.118^{*}$ & -.061 & $.325^{* *}$ & 1.000 & & & \\
\hline & .005 & .041 & .292 & .000 & & & & \\
\hline \multirow{2}{*}{ Hiperactividad } & $-.177^{* *}$ & .091 & -.011 & -.108 & $.260^{* *}$ & 1.000 & & \\
\hline & .002 & .116 & .843 & .062 & .000 & & .000 & \\
\hline \multirow{2}{*}{$\begin{array}{l}\text { Problemas } \\
\text { compañeros }\end{array}$} & $-.200^{* *}$ & .108 & .001 & $-.122 *$ & $.391^{* *}$ & $.445^{* *}$ & 1.000 & \\
\hline & .001 & .061 & .985 & .034 & .000 & .000 & & \\
\hline \multirow{2}{*}{$\begin{array}{l}\text { Conducta } \\
\text { prosocial }\end{array}$} & .094 & $-.141^{*}$ & .017 & .098 & $-.298^{* *}$ & $-.291^{* *}$ & $-.432^{* *}$ & 1.000 \\
\hline & .103 & .015 & .772 & .090 & .000 & .000 & .000 & \\
\hline
\end{tabular}

${ }^{\star \star}$ La correlación es significativa al nivel .001 (bilateral); ${ }^{\star}$ La correlación es significativa al nivel .05 (bilateral)

Fuente: elaboración propia

3.3. Dificultades emocionales y de comportamiento, procesos de comunicación, vocabulario receptivo y razonamiento abstracto de niños y adolescentes.

Respecto al segundo objetivo, la puntuación total de SDQ de los niños y adolescentes fue pequeña y significativamente correlacionada con los profesores especialistas $(r=.250, p<.001)$ y tutores $(r=.247, p$ $<.001$ ) (Tabla 2). Se documentaron los vínculos entre las dificultades emocionales y de comportamiento de los niños y adolescentes y su vocabulario receptivo. Los análisis de correlación indicaron que la puntuación total de SDQ de los profesores especialistas fue significativa, pequeña y correlacionada negativamente con la puntuación final de PPVT de los niños y adolescentes $(r=-.113, p<.05)$, ya que cuando la puntuación total de los profesores especialistas aumentaba, el puntuación final de PPVT disminuía en una proporción constante. Además, los análisis de correlación mostraron que los valores de los especialistas fueron pequeños y estaban significativamente asociados con los de los tutores $(r=.258, p<.001)$ en la puntuación total de SDQ. 
Tabla 2

Correlaciones entre las puntuaciones totales finales del SDQ, ITPA, PPVT y RPM para profesorado y niños y adolescentes con dispositivos auditivos

\begin{tabular}{lcccccc}
\hline & SDQPE & SDQPT & SDQNI & ITPANI & PPVTNI & RPMNI \\
\hline SDQPE & 1.000 & & & & & \\
SDQPT & $.258^{* *}$ & 1.000 & & & & \\
SDQNI & $.250^{* *}$ & $.247^{* *}$ & 1.000 & & & \\
ITPANI & .053 & .037 & $.115^{*}$ & 1.000 & 1.000 & \\
PPVTNI & $-.113^{*}$ & .050 & -.062 & -.073 & .077 & 1.000 \\
RPMNI & .032 & .053 & .053 & $.115^{*}$ & .000 \\
\hline
\end{tabular}

${ }^{* *} p<.010 ;{ }^{*} p<.00$

SDQPE:SDQ Profesorado especialista en audición y lenguaje; SDQPT:SDQ Profesorado tutor; SDQNI:SDQ Niños que usan dispositivos auditivos; ITPANI:ITPA Niños que usan dispositivos auditivos; PPVTNI:PPVT Niños que usan dispositivos auditivos; RPMNI:RPM Niños que usan dispositivos auditivos.

Fuente: elaboración propia

Como se indica en la Tabla 3, las subescalas del ITPA correlacionaron significativamente entre sí (p. ej., Comprensión Auditiva con Expresión Verbal $(r=.616, p<.001)$, Comprensión Auditiva con Memoria Auditiva $(r=.632, p<.001)$, y Comprensión Auditiva con Integración Gramatical $(r=.581, p<.001)$. Estas correlaciones mostraron una asociación entre dos niveles de organización de complejidad creciente. Las correlaciones entre las subescalas de ITPA y las de SDQ son muy bajas o no hay correlación, lo que refleja que cada prueba es independiente y mide aspectos claramente diferentes.

Tabla 3

Correlaciones entre las subescalas de SDQ y del ITPA en niños y adolescentes

\begin{tabular}{|c|c|c|c|c|c|c|c|c|c|c|c|c|c|c|c|}
\hline & $\begin{array}{c}\text { SDQ } \\
1\end{array}$ & $\begin{array}{c}\text { SDQ } \\
2 \\
\end{array}$ & $\begin{array}{c}\text { SDQ } \\
3\end{array}$ & $\begin{array}{c}\mathrm{SDQ} \\
4\end{array}$ & $\begin{array}{c}\text { SDQ } \\
5\end{array}$ & $\begin{array}{c}\text { ITPA } \\
1 \\
\end{array}$ & $\begin{array}{c}\text { ITPA } \\
2 \\
\end{array}$ & $\begin{array}{c}\text { ITPA } \\
3 \\
\end{array}$ & $\begin{array}{c}\text { ITPA } \\
4 \\
\end{array}$ & $\begin{array}{c}\text { ITPA } \\
5 \\
\end{array}$ & $\begin{array}{c}\text { ITPA } \\
6 \\
\end{array}$ & $\begin{array}{c}\text { ITPA } \\
7 \\
\end{array}$ & $\begin{array}{c}\text { ITPA } \\
8 \\
\end{array}$ & $\begin{array}{c}\text { ITPA } \\
9 \\
\end{array}$ & $\begin{array}{c}\text { ITPA1 } \\
0 \\
\end{array}$ \\
\hline SDQ1 & 1.000 & & & & & & & & & & & & & & \\
\hline SDQ2 & $\underset{* *}{283}$ & 1.000 & & & & & & & & & & & & & \\
\hline SDQ3 & $\underset{\star \star}{-.232}$ & .226 & 1.000 & & & & & & & & & & & & \\
\hline SDQ4 & $-\underset{\star *}{207}$ &.$_{\star \star \star}^{288}$ & .473 & 1.000 & & & & & & & & & & & \\
\hline SDQ5 & .151 & $\underset{* \star \star}{-334}$ & -.327 & $\underset{\star \star \star}{-386}$ & 1.000 & & & & & & & & & & \\
\hline ITPA1 & $\underset{*}{.137}$ & -.012 & -.003 & -.051 & .031 & 1.000 & & & & & & & & & \\
\hline ITPA2 & .101 & -.063 & $\begin{array}{c}-.149 \\
*\end{array}$ & $\underset{*}{-.150}$ & .130 & .230 & 1.000 & & & & & & & & \\
\hline ITPA3 & .047 & .081 & .117 & .105 & -.099 & $\begin{array}{c}.616 \\
\star \star \star\end{array}$ & .023 & 1.000 & & & & & & & \\
\hline ITPA4 & -.044 & .003 & -.032 & -.064 & .002 & -.033 & .305 & -.133 & 1.000 & & & & & & \\
\hline ITPA5 & -.020 & .054 & .029 & .006 & -.048 & .268 & .114 & .302 & $\underset{* \star \star}{.241}$ & 1.000 & & & & & \\
\hline ITPA6 & .047 & -.050 & -.078 & -.093 & .066 & .052 & .635 & .019 & $\begin{array}{c}.467 \\
\star \star \star\end{array}$ & .068 & 1.000 & & & & \\
\hline ITPA7 & .023 & .082 & .060 & -.012 & -.081 & $\underset{* * *}{.632}$ & ${ }_{* \star}^{.188}$ & .486 & -.102 & $\underbrace{.242}_{* \star *}$ & .035 & 1.000 & & & \\
\hline ITPA8 & $\begin{array}{c}.149 \\
*\end{array}$ & .004 & -.079 & -.103 & .141 & .581 & $\underset{* * *}{.576}$ & $\underset{\star \star * \star}{.462}$ & $\underset{* *}{.218}$ & $\underset{* * *}{.300}$ & .457 & $\begin{array}{c}.435 \\
\star * \star\end{array}$ & 1.000 & & \\
\hline ITPA9 & $\underset{*}{169}$ & -.130 & -.130 & -.103 & .129 & .309 & .055 & $\begin{array}{l}.324 \\
* \star \star\end{array}$ & -.064 & -.028 & $\begin{array}{c}149 \\
*\end{array}$ & $\underset{* *}{215}$ & .208 & 1.000 & \\
\hline $\begin{array}{c}\text { ITPA1 } \\
0\end{array}$ & .129 & .047 & -.103 & $\underset{*}{-.136}$ & .055 & .003 & .277 & -.042 & .097 & -.060 & $\underset{* \star}{218}$ & -.010 & .250 & -.127 & 1.000 \\
\hline
\end{tabular}


SDQ1:Problemas emocionales; SDQ2:Problemas conductuales; SDQ3:Hiperactividad; SDQ4:Problemas con los compañeros; SDQ5:Conducta prosocial.

ITPA1:Nivel Representativo:Auditivo-Vocal Comprensión Auditiva; ITPA2:Nivel Representativo:Auditivo-Vocal Asociación Auditiva; ITPA3:Nivel Representativo:Auditivo-Vocal Expresión Verbal; ITPA4:Nivel Representativo Viso-Motor Comprensión Visual; ITPA5:Nivel Representativo Viso-Motor AsociaciónVisual; ITPA6:Nivel Representativo Viso-Motor Expresión Motora; ITPA7:Nivel Automático Auditivo-Vocal Memoria Secuencial Auditiva; ITPA8:Nivel Automático AuditivoVocal Integración Gramatical; ITPA9:Nivel Automático Auditivo-Motor Memoria Secuencial Visomotora; ITPA10:Nivel Automático Auditivo-Vocal Integración Auditiva.

Fuente: elaboración propia

3.4. Análisis del efecto causal de las variables sociodemográficas en la puntuación total de SDQ.

Para analizar el tercer objetivo se realizó una regresión lineal buscando una $R_{2}$ tan cerca de 1 que indicara un ajuste lineal perfecto. No se obtuvo un porcentaje de varianza explicado que fuera destacado para los tests PPVT y SPM.

En el caso de la puntuación total de SDQ y empleando variables sociodemográficas como predictoras, se empleó el método de introducción del análisis de regresión lineal múltiple, que cumplió los supuestos de linealidad, independencia, normalidad, homoscedasticidad y no colinealidad para garantizar la validez del modelo.

Se eligió el método de mínimos cuadrados para averiguar su función continua (Tabla 4). El coeficiente de correlación de Pearson fue .751, mientras que la bondad del ajuste se calculó siguiendo el coeficiente de determinación $R_{2}$, cuyos valores oscilaron entre -1 y 1 . Por lo tanto, el valor corregido de $R_{2}=.551$ predijo $55.1 \%$ de la varianza (efecto corregido de la muestra y variables independientes). El error de estimación típico (raíz cuadrada de la varianza inexplicada) resultó 0.468 . La prueba ANOVA mostró que la varianza explicada por la regresión fue significativamente diferente (y superior) a la varianza inexplicada. El modelo fue $F=$ $42.431, p<.001$, que significó un efecto real de estas variables en la puntuación total de SDQ de los niños y adolescentes con dispositivos auditivos.

Finalmente, el modelo reflejó que las variables independientes (p. ej., dispositivo auditivo, ubicación geográfica, $\mathrm{ACl}$, edad de las madres y edad cronológica de los niños) fueron estadísticamente significativas, siendo el dispositivo auditivo (ICs/AUDs) el coeficiente más alto $(t=10,511, p<.000)$.

Tabla 4.

Análisis de regresión de las variables sociodemográficas como predictores de la puntuación total de SDQ de los niños y adolescentes

\begin{tabular}{|c|c|c|c|c|c|c|c|}
\hline $\begin{array}{l}\text { Modelo } \\
\text { regresión }\end{array}$ & $\mathrm{R}$ & $\mathrm{R}_{2}$ & $F$ & $\begin{array}{l}\text { Varianza } \\
\text { explicada }\end{array}$ & $\begin{array}{l}\text { Predictores socio- } \\
\text { demográficos }\end{array}$ & $\beta$ & $t$ \\
\hline $\begin{array}{l}\text { Puntuación } \\
\text { total niños } \\
\text { SDQ }\end{array}$ & .751 & .564 & $42.431^{\star \star \star}$ & $55.1 \%$ & $\begin{array}{c}\text { Dispositivo auditivo } \\
\text { (IC/AUD) } \\
\text { Localización geográfica } \\
\text { Adaptación curricular (ACI) } \\
\text { Edad cronológica de la } \\
\text { madre } \\
\text { Edad cronológica de los } \\
\text { niños }\end{array}$ & $\begin{array}{c}1.155 \\
-.268 \\
.230 \\
-.1504 \\
.078\end{array}$ & $\begin{array}{c}10.511^{\star * *} \\
-3.278^{\star * *} \\
3.019^{\star *} \\
-2.789^{\star *} \\
2.006^{\star}\end{array}$ \\
\hline
\end{tabular}

${ }^{* *} p<.001 ;{ }^{* *} p<.010 ;{ }^{*} p<.00$

3.5. Análisis del efecto causal de variables de áreas de ITPA en la puntuación total de SDQ.

Los resultados del análisis de regresión se muestran en la Tabla 5, donde algunas subescalas de ITPA explicaron $42.6 \%$ de la varianza de la puntuación total de SDQ de los niños y adolescentes. El Nivel Automático Auditivo-Vocal tuvo un peso importante en la puntuación de las fortalezas y debilidades de la puntuación total de SDQ de los niños, específicamente, las escalas Memoria Secuencial Auditiva, y con un valor significativo aún mayor, Integración Gramatical $(t=3.781, p<.001)$. En el Nivel Representativo AuditivoVocal la escala Expresión Verbal obtuvo un destacado nivel de significación y Viso-Motor en la escala de Comprensión Visual, igualmente tuvo un peso significativo.

El valor $F$ obtenido fue 8.594, que significó un efecto real de estas variables en la puntuación total de SDQ de los niños y adolescentes.

Se puede afirmar que la puntuación total de SDQ estuvo claramente influenciada por los hábitos integrados, como la memoria y el aprendizaje remoto, que producen una cadena automática de respuestas, así como las 
modalidades visuales o auditivas que constituyen la entrada del canal de comunicación, resaltando la influencia más alta del Nivel Automático Auditivo-Motor en la Memoria Secuencial Visomotora $(t=4.502, p<$ $.001)$.

Tabla 5.

Análisis de regresión de las variables ITPA, Peabody y Raven como predictoras de la puntuación total de SDQ de los niños y adolescentes

\begin{tabular}{|c|c|c|c|c|c|c|c|}
\hline $\begin{array}{l}\text { Modelo } \\
\text { regresión }\end{array}$ & $\mathrm{R}$ & $\mathrm{R}_{2}$ & $\mathrm{~F}$ & $\begin{array}{l}\text { Varianza } \\
\text { explicada }\end{array}$ & Predictores socio-demográficos & $\beta$ & $t$ \\
\hline $\begin{array}{l}\text { Puntuación } \\
\text { total niños } \\
\text { SDQ }\end{array}$ & .426 & .181 & $\underset{\star \star \star}{8.594}$ & $42,6 \%$ & $\begin{array}{c}\text { Nivel Automático Auditivo-Motor Memoria } \\
\text { Secuencial Visomotora } \\
\text { Nivel Automático Auditivo-Vocal Memoria } \\
\text { Secuencial Auditiva Nivel Automático Auditivo- } \\
\text { Vocal Integración Gramatical } \\
\text { Nivel Representativo Auditivo-Vocal Expresión } \\
\text { Verbal } \\
\text { Nivel Representativo Viso-Motor Comprensión } \\
\text { Visual }\end{array}$ & $\begin{array}{l}.022 \\
-.014 \\
.015 \\
-.014 \\
-.014\end{array}$ & $\begin{array}{l}4.502^{\star \star *} \\
-2.934^{\star *} \\
3.781^{\star * *} \\
-2.846^{\star *} \\
-2.683^{\star *}\end{array}$ \\
\hline
\end{tabular}

${ }^{* \star *} p<.001 ;{ }^{* *} p<.010 ;{ }^{*} p<.00$

\section{Discusión}

\subsection{Medición de acuerdos entre evaluadores de subescalas SDQ.}

El estudio ha investigado patrones de acuerdo entre padres, profesorado y niños y adolescentes en las subescalas de SDQ. Se utilizó el coeficiente kappa de Cohen de medidas categóricas para estudiar la correspondencia entre los informantes que calificaron los problemas y dificultades de los niños y adolescentes de manera discrepante. Sin embargo, ha habido pares de informantes que causaron diferentes acuerdos en los coeficientes kappa de Cohen (p. ej., padres-niños y adolescentes con ICs, profesor tutor-padres, tutoresniños y adolescentes con ICs). Las evaluaciones de niños y adolescentes han originado niveles de correspondencia bajos a moderados entre los informantes produciéndose niveles bajos de correspondencia cuando los informantes han tenido oportunidades relativamente más importantes para observar (p. ej., los especialistas han percibido más problemas de conducta en los niños y adolescentes que usaban AUDs) dentro del mismo contexto de clase, mientras que el profesorado tutor ha destacado más los síntomas emocionales de los niños y adolescentes que usaban ICs.

Se ha considerado que los padres tenían una posición única para observar a los niños y adolescentes en un gran número de circunstancias y durante largos períodos de tiempo con respecto a otros informantes, como era el profesorado. Sin embargo, la baja o nula concordancia entre los niños y adolescentes y sus padres en este estudio se ha debido probablemente a la incapacidad o a la negativa de los niños y adolescentes a informar de sus verdaderos problemas a sus padres. En este sentido, las familias suelen tener un conocimiento limitado sobre las relaciones interpersonales de los niños y adolescentes con otros compañeros (McAloney-Kocaman y McPherson, 2017).

4.2. Relación entre dificultades emocionales y de comportamiento, procesos de comunicación, el vocabulario receptivo y el razonamiento abstracto de niños y adolescentes con dispositivos auditivos. En general, los problemas de aprendizaje implican muchos factores psicológicos encubiertos. En efecto, las puntuaciones de la subescala Problemas con los compañeros fueron significativos y se asociaron negativamente con la Conducta Prosocial, significando con ello que la amistad entre los niños y adolescentes se desarrollaba mientras disminuían los Problemas con los compañeros (Durkin y Conti-Ramsden, 2007). Los participantes que se evaluaron a sí mismos más alto en la Conducta Prosocial, calificaron inversamente los Problemas de conducta, la Hiperactividad y los Problemas con sus compañeros.

Este resultado se puede deber a la submuestra de adolescentes del estudio, cuya Conducta Prosocial, especialmente hacia los amigos, constituye una característica clave de la edad. Las correlaciones de los niños y adolescentes con pérdida auditiva entre las subescalas de SDQ no fueron elevadas revelando así diferencias entre las subescalas de SDQ según factores distales (p. ej., se había administrado el mismo SDQ a diferentes padres y/o madres, profesorado y a estudiantes de diferentes grados y provincias). Además, los resultados han ocultado que los niños y adolescentes con ICs o AUDs son iguales antes de la colocación del 
dispositivo auditivo, debido a la falta de datos de referencia. Esta limitación se ha subrayado igualmente en otros estudios (Jiménez, Pino y Herruzo, 2009).

Las correlaciones entre las puntuaciones de ITPA y SDQ han mostrado una relación baja, como se ha observado anteriormente, donde ITPA ha mantenido correlaciones bajas con otras pruebas (Kirk y Elkins, 1974). Sin embargo, las tres subpruebas auditivas de ITPA estaban relacionadas entre sí. Las subpruebas Asociación auditiva y Expresión verbal se han utilizado para la selección y reagrupamiento de niños y adolescentes para el tratamiento de adaptaciones curriculares individualizadas (ACl). Además, estas subpruebas de representación están significativamente correlacionadas con las matrices progresivas de Raven (Kirk y Elkins, 1974).

En los escolares con dispositivos auditivos, la necesidad de observación continua de su pérdida auditiva y la complejidad para identificar sus problemas sociales y de comunicación dificultan el diagnóstico basado en informes del profesorado, como han reportado otros autores (Henner, Novogrodsky, Reis y Hoffmeister, 2018).

\subsection{Predictores de dificultades emocionales y de comportamiento}

a) Dispositivos auditivos. Los niños y adolescentes que han empleado ICs o AUDs han mostrado desacuerdos en las puntuaciones de las subescalas SDQ. No obstante, el primer factor que ha entrado en el modelo de regresión causando el resultado total de SDQ ha sido la presencia de niños y adolescentes con ICs o AUDs en las aulas (Sarant, Harris, Bennet y Bant, 2014).

b) Localización geográfica. El modelo de regresión ha incorporado predictores sociodemográficos de la puntuación total de SDQ. La provincia ha sido un predictor de las dificultades emocionales y de comportamiento de los niños y adolescentes, como ocurrió en el estudio de Neuss (2006). El modelo de prestación de servicios de la provincia de Gran Canaria es diferente del modelo de la provincia de Tenerife, ya que en el primer caso cuenta con el Hospital Universitario Insular Materno-Infantil de Canarias, donde se llevan a cabo los implantes y posee recursos técnicos y personales. Como implicación, sería interesante replicar este estudio en otras provincias españolas donde se empleen modelos similares o diferentes.

c) $A C l$. El tratamiento educativo $\mathrm{ACl}$ ha sido una causa del resultado total de SDQ. La ruta del currículo adaptado que ha tomado cada estudiante ha dependido del diagnóstico de sus habilidades en el ITPA, del aprendizaje de memoria y de las habilidades integradas.

d) Edad de la madre. Este estudio ha subrayado la edad de las madres como un predictor para resolver problemas de niños y adolescentes con dispositivos auditivos. En el futuro, se deberá investigar los beneficios de la educación materna y el apoyo de las madres a otras madres como reportaron Henderson, Johnson y Moodie (2014).

e) Edad cronológica de los niños. Según nuestro estudio y el de otros investigadores, la edad del niño influye en las dificultades emocionales y de comportamiento (Asker-Arnason, Ibertsson, Wass, Wengelin y Sahlen, 2010). De ello se colige que las futuras investigaciones deberán centrarse en la audición funcional que tienen los niños y adolescentes desde el nacimiento hasta que reciben sus implantes o audífonos y desarrollan la comunicación oral.

f) Subescala ITPA. El análisis de regresión de este estudio ha mostrado que el factor ITPA de nivel Automático Auditivo-Vocal en la escala de Memoria Secuencial Visomotora predice la puntuación total de SDQ en los niños y adolescentes con dispositivos auditivos. Su dificultad para obtener un significado del material presentado oralmente y evaluar su conocimiento semántico les causa problemas en la percepción de sus fortalezas y debilidades emocionales. Consecuentemente, se precisa una interacción sensible entre especialistas, tutores, familias y los propios niños y adolescentes con dispositivos auditivos para lograr superar las barreras que les impiden alcanzar adecuados niveles comunicativos y de razonamiento abstracto, así como de desarrollo emocional. 


\section{Limitaciones e Implicaciones educativas}

El presente estudio tiene algunas limitaciones ya que los resultados solo son propios de la mayoría de la población de niños con ICs y AUDs de Islas Canarias, sin otros déficits asociados. Además, se precisa evaluar otros factores del entorno familiar, del tipo de pérdida auditiva o prototipos de dispositivos que no se han evaluado en plenitud en el presente estudio.

Los desacuerdos en las valoraciones entre el profesor especialista y el profesor tutor de aula denotan una falta de conocimiento de los profesionales de la educación de la realidad de los niños y adolescentes del presente estudio. Por consiguiente, los programas de formación del profesorado han de incorporar aspectos curriculares relativos a un mejor conocimiento de las dificultades emocionales y de comportamiento, los procesos de comunicación, el vocabulario receptivo y el razonamiento abstracto de estudiantes que usan ICs y AUDs, y programar una mejor coordinación entre los distintos profesionales (Henner, Novogrodsky, Reis y Hoffmeister, 2018). Este estudio sugiere, asimismo, la necesidad de asesorar a los familiares, debido a la poca concordancia entre los niños y adolescentes que usan ICs y AUDs y sus familias (Archbold, Sach, O'neill, Lutman, y Gregory, 2008).

\section{Conclusiones}

Los resultados de este estudio muestran que los niños y adolescentes con ICs y AUDs, sus padres y el profesorado evaluaron de manera divergente las dificultades emocionales y de comportamiento de los niños y adolescentes con ICs y AUDs. Aunque el estudio encontró pruebas de que SDQ e ITPA no estaban correlacionados, se revelaron correlaciones significativas entre algunas subescalas de ITPA. El estudio identificó factores predictivos de los resultados en esta población estudiantil (p. ej., tipo de dispositivo auditivo, ubicación geográfica, adaptación curricular individualizada, edad de las madres, edad cronológica de los niños y algunas subescalas de ITPA). Los hallazgos tienen consecuencias para el desarrollo de programas formativos del profesorado de educación primaria y secundaria, así como en los sistemas evaluativos relacionados con los aspectos emocionales y de comportamiento, los procesos de comunicación, el vocabulario receptivo y el razonamiento abstracto empleados con los niños y adolescentes que usan dispositivos auditivos en Islas Canarias.

\section{Referencias}

Archbold, S., Sach, T., O'neill, C., Lutman, M., y Gregory, S. (2008). Outcomes From Cochlear Implantation for Child and Family: Parental Perspectives. Deafness \& Education International, 10(3), 120-142. https://doi:

$10.1179 / 146431508790559751$

Asker-Arnason, L., Ibertsson, T., Wass, M., Wengelin, A., y Sahlen, B. (2010). Picture-Elicited Written Narratives, Process and Product, in 18 Children with Cochlear Implants. Communication Disorders Quarterly, 31(4), $195-212$. https://doi.org/10.1177/1525740109337734.

Bilker, W.B., Hansen, J.A., Brensinger, C.M., Richard, J., Gur, R.E., y Gur, R.C. (2012). Development of Abbreviated Nine-Item Forms of the Raven's Standard Progressive Matrices Test. Assessment 19(3), 354-369. https://doi:10.1177/1073191112446655.

Ching, T.Y.C., Scarinci, N., Marnane, V., Sjahalam-King, J., Button, L., y Whitfield, J. (2018). Factors influencing parents' decisions about communication choices during early education of their child with hearing loss: a qualitative study. Deafness \& Education International. https://doi.org/10.1080/14643154.2018.1512393.

Compton, M.V., Tucker, D.A., y Flynn, P.F. (2009). Preparation and Perceptions of Speech-Language Pathologists Working With Children With Cochlear Implants. Communication Disorders Quarterly, 30(3), 142-154. https://doi:142154.10.1177/1525740108325554.

Connor, C. M., Craig, H. K., Raudenbush, S. W., Heavner, K., y Zwolan, T. A. (2006). The age at which young children receive cochlear implants and their vocabulary and speechproduction growth: Is there an added value for early implantation? Ear and Hearing, 27, 628-644. https://doi:0196/0202/06/2706-0628/0.

De Los Reyes, A., y Kazdin, A. (2005). Informant discrepancies in assessment of childhood psychopathology: A critical review. Psychological Bulletin, 131, 483-509.

Dunn, M., y Dunn, L.M. (2007). Peabody Picture Vocabulary Test-4. Circle Pines, MN: AGS.

Durkin, K., y Conti-Ramsden, G. (2007). Language, social behavior, and the quality of friendships in adolescents with and without a history of specific language impairment. Child Dev., 78(5), 1441-57. https://doi: 10.1111/j.14678624.2007.01076.x.

Edwards, L., Hill, T., y Mahon, M. (2012). Quality of life in children and adolescents with cochlear implants and additional needs. International Journal of Pediatric Otorhinolaryngology, 76(6), 851-857. https://doi:10.1016/j.ijporl.2012.02.057. Eriks-Brophy, A., y Whittingham, J. (2013). Teachers' perceptions of the inclusion of children with hearing loss in general education settings. American Annals of the Deaf, 158(1), 63-97.

George, D., y Mallery, M. (2003). Using SPSS for Windows step by step: a simple guide and reference. Allyn \& Bacon. 
Goodman, R. (1997). The strengths and difficulties questionnaire: a research note. J. Child. Psychol. Psychiatry, 38(5), 581-586.

Goodman, R. (2001). Psychometric properties of the Strengths and Difficulties Questionnaire. Journal of the American Academy of Child \& Adolescent Psychiatry, 40, 1337-1345. https://doi:10.1097/00004583-200111000-00015. Gresham, F.M., Elliott, S.N., Metallo, S., Byrd, S., Wilson, E., y Cassidy, K. (2017). Cross-informant agreement of children's social-emotional skills: An investigation of ratings by teachers, parents, and students from a nationally representative sample. Psychology in the Schools, 55(2). https://doi.org/10.1002/pits.22101. Hassan, H.E., Eeldin, S.T.K., y Al Kasaby, R.M. (2014). Psycholinguistic abilities in cochlear implant and hearing impaired children. Egyptian Society of Ear, Nose, Throat and Allied Sciences, 15, 29-35. https://doi.org/10.1016/j.ejenta.2013.12.006.

Henderson, R.J., Johnson, A., y Moodie, S. (2014). Parent-to-Parent Support for Parents With Children Who Are Deaf or Hard of Hearing: A Conceptual Framework. Journal of Audiology (Online), 23(4), 437-438. https://doi:10.1044/2014_AJA14-0029.

Henner, J., Novogrodsky, R., Reis, J., y Hoffmeister, R. (2018). Recent Issues in the use of Signed Language Assessments for Diagnosis of Language Disorders in Signing Deaf and Hard of Hearing children. Journal of Deaf Studies and Deaf Education, 1-10. https://doi:10.1093/deafed/eny014.

Huber, M., y Kipman, U. (2011). The mental health of deaf adolescents with cochlear implants compared to their hearing peers. International Journal of Audiology, 50, 146-154. https://doi:10.310.9/14992027.2010.533704.

Isarin, J., Zadelhoff, I., Wolters-Leermakers, N., Speksnijder-Bregman, M., Hannink, M., y Knoors, H. (2015). A World of Difference. Parental Perspectives on Cochlear Implantation in Deaf Children with Additional Disabilities. Deafness \& Education International, 17(4), 219-230. https://doi: 10.1179/1557069X15Y.0000000007.

Jiménez, M.S., Pino, M.J., y Herruzo, J. (2009). A comparative study of speech development between deaf children with cochlear implants who have been educated with spoken or spoken+sign language. Revista Internacional de Otorrinolaringología Pediátrica, 73(1), 109-14. https://doi:10.1016/j.ijporl.2008.10.007.

Kirk, S.A., y Elkins, J. (1974). Summaries of Research on the Revised Illinois Test of Psycholinguistics. Final Report. ERIC (ED115024).

Kirk, S.A., McCarthy, J.J., y Kirk, W.D. (2001). Illinois Test of Psycholinguistic Abilities. Madrid, Spain: TEA. Laugen, N.J., Jacobsen, K.H., Rieffe, C., y Wichstrøm, L. (2017). Social skills in preschool children with unilateral and mild bilateral hearing loss. Deafness \& Education International, 19(2), 54-62.

https://doi:10.1080/14643154.2017.1344366.

Lúcio, P.S., Cogo-Moreira, H., Puglisi, M., Polanczyk, G.V., y Little, T.D. (2017). Psychometric Investigation of the Raven's Colored Progressive Matrices Test in a Sample of Preschool Children. Assessment, Nov., 1-11. https://doi:10.1177/1073191117740205.

McAloney-Kocaman, K., y McPherson, K. (2017). Factor Structure and Reliability of the Parent-Informant Strengths and Difficulties Questionnaire in a Scottish Preschool Sample. Early Education and Development, 28(3), 368-376. https://doi:10.1080/10409289.2017.1228367.

Neuss, D. (2006). The ecological transition to auditory-verbal therapy: Experiences of parents whose children use cochlear implants. The Volta Review, 106(2), 195-222.

Ortuño-Sierra, J., Aritio-Solana, R., y Fonseca-Pedrero, E. (2018). Health difficulties in children and adolescents: The study of the SDQ in the Spanish National Health Survey 2011-2012. Psychiatry Research, 259, 236-

242, https://doi.org/10.1016/j.psychres.2017.10.025.

Ortuño-Sierra, J., Fonseca-Pedrero, E., Inchausti, F., y Sastre i Riba, S. (2016). Evaluación de dificultades emocionales y comportamentales en población infanto-juvenil: El Cuestionario de Capacidades y Dificultades (SDQ). Papeles del Psicólogo, 37(1), 14-26.

Peng, C-Y.P., y Chen, L-T. (2014). Beyond Cohen's d: Alternative Effect Size Measures for Between-Subject Designs. The Journal of Experimental Education, 82(1), 22-50. https://doi:10.1080/00220973.2012.745471.

Porter, H., Sladen, D.P., Ampah, S.B., Rothpletz, A., y Bess, F.H. (2013). Developmental outcomes in early school-age children with minimal hearing loss. American journal of audiology, 22(2), 263-270. https://doi:10.1044/10590889(2013/13-0013.

Raven, J. (1989). The Raven Progressive Matrices: A review of national norming studies and ethnic and socioeconomic variation within the United States. Journal of Educational Measurement, 26, 1-16. https://doi.org/10.1111/j.1745-3984. Raven, J. (2000). The Raven's Progressive Matrices: Change and stability over culture and time. Cognitive Psychology, 41, 1-48. https://doi.org/10.1006/cogp.1999.0735.

Rodríguez-Hernández, P.J., Betancort, M., Ramírez-Santana, G.M., García, R., Sanz-Alvarez, E.J., y De las CuevasCastresana, C. (2014). Puntos de corte de la versión española del Cuestionario de Cualidades y Dificultades (SDQ). Revista de Psiquiatría Infanto-Juvenil, 3, 23-29.

Sarant, J., Harris, D., Bennet, L., y Bant, S. (2014). Bilateral Versus Unilateral Cochlear Implants in Children: A Study of Spoken Language Outcomes. Ear and Hearing, 35(4), 396-409. https://doi:10.1097/AUD.0000000000000022.

Sarchet, T., Marschark, M., Borgna, G., Convertino, C., Sapere, P., y Dirmyer, R. (2014). Vocabulary Knowledge of Deaf and Hearing Postsecondary Students. J Postsecond Educ Disabil, 27(2), 161-

178. https://doi:10.1016/j.ijporl.2008.06.016. 
Shirvani, S., Jafari, Z., Zarandi, M.M., Jalaie, S., Mohagheghi, H., y Tale, M.R. (2016). Emotional Perception of Music in Children With Bimodal Fitting and Unilateral Cochlear Implant. Annals of Otology, Rhinology \& Laryngology, 125(6), 470477. https://doi:10.1177/0003489415619943.

Wong, C. L., Ching, T.Y.C., Cupples, L., Button, L., Leigh, G., Marnane, V. ... Martin, L. (2017). Psychosocial Development in 5-Year-Old Children With Hearing Loss Using Hearing Aids or Cochlear Implants. Trends in Hearing, 21, 1-19. https://doi:10.1177/2331216517710373.

Wong, C. L., Ching, T.Y.C., Cupples, L., Leigh, G., Marnane, V., Button, L. ... Gunnourie, M. (2018). Comparing parent and teacher ratings of emotional and behavioural difficulties in 5-year old children who are deaf or hard-of-hearing. Deafness \& Education International, Published Online: 24 May: 1-24. https://doi.org/10.1080/14643154.2018.1475956. 